\title{
Partos domiciliares planejados na região de Campinas de 2013 a 2017
}

\author{
Planned home births in the region of Campinas from 2013 to 2017 \\ Partos domiciliarios planificados en la región de Campinas de 2013 a 2017
}

Recebido: 08/09/2021 | Revisado: 15/09/2021 | Aceito: 18/09/2021 | Publicado: 18/09/2021

Fernanda de Almeida Silveira

ORCID: https://orcid.org/0000-0002-6360-7550

Universidade Brasil, Brasil

Faculdade São Leopoldo Mandic, Brasil E-mail: fersilveira39@yahoo.com.br

Adriana de Lima Mello

ORCID: https://orcid.org/0000-0002-1169-9609 Enfermeira obstetra autônoma, Brasil

E-mail: adrianadelimamello@gmail.com

Camila Claro de Castro

ORCID: https://orcid.org/0000-0003-2682-8888

Enfermeira obstetra autônoma, Brasil

E-mail: camilinhaclaro@gmail.com

Alessandra Baptista

ORCID: https://orcid.org/0000-0003-1591-7009

Universidade Brasil, Brasil

E-mail: alessandra.baptista@universidadebrasil.edu.br

Amanda Farage Frade Barros

ORCID: https://orcid.org/0000-0002-9004-8811

Universidade Brasil, Brasil

E-mail: amanda.frade@universidadebrasil.edu.br

Silvia Cristina Nunez

ORCID: https://orcid.org/0000-0003-2101-8333

Universidade Brasil, Brasil

E-mail: silvia.nunez@universidadebrasil.edu.br

Clara Fróes de Oliveira Sanfelice

ORCID: https://orcid.org/0000-0003-1920-3193

Universidade Estadual de Campinas, Brasil

E-mail: clara_sanfelice@yahoo.com.br

Daniel Souza Ferreira Magalhães

ORCID: https://orcid.org/0000-0002-3761-1927

Universidade Brasil, Brasil

E-mail: daniel.magalhaes@universidadebrasil.edu.br

\section{Resumo}

Nas últimas décadas tem-se observado um número crescente de nascimentos que são assistidos por profissionais de saúde habilitados e que são planejados antecipadamente para acontecerem em domicílio. Estes têm sido alvo de discussões na mídia, nas redes sociais, entre os conselhos profissionais de saúde e entre os mais diversos setores da sociedade. Neste estudo retrospectivo quantitativo e descritivo, temos por objetivo analisar e descrever os resultados dos partos domiciliares planejados atendidos por uma equipe de enfermeiras obstetras da cidade de Campinas, Brasil no período de 2013 a 2017. Os prontuários analisados eram de mulheres de risco obstétrico habitual, que tiveram parto domiciliar planejado atendido por uma equipe de enfermeiras obstétricas $(n=160)$. Os dados foram obtidos por análise dos prontuários das parturientes e as variáveis analisadas foram os dados sóciodemográficos, o perfil obstétrico, os resultados obstétricos e neonatais. Os resultados mostraram que 68,75\% dos partos tiveram duração de até 8 horas, $53,44 \%$ das parturientes não tiveram lacerações de períneo durante o período expulsivo, a taxa de transferência materna pós-parto foi de $2,52 \%$ e apenas $18,81 \%$ das parturientes necessitaram de intervenção durante o processo do parto. Em relação aos neonatais, 97,41\% tiveram Apgar de $1^{\circ}$ minuto acima de 8 e $100 \%$ tiveram Apgar de $5^{\circ}$ minuto acima de 8 . Verificamos que os resultados são compatíveis com estudos internacionais que consideram o parto domiciliar planejado tão seguro quanto o hospitalar, além de comprovar a utilização dos preceitos da humanização ao parto, que respeita as escolhas da mulher durante o processo fisiológico de parir. Pela análise dos resultados e comparação com dados da literatura, podemos concluir que o parto domiciliar planejado com mulheres de risco obstétrico habitual não oferece riscos adicionais ao parto tradicional em hospital, tanto para a parturiente quanto para o bebê.

Palavras-chave: Parto domiciliar; Humanização de assistência ao parto; Sistemas de informação em saúde; Bioengenharia. 


\begin{abstract}
In recent decades, there has been an increasing number of births that are assisted by qualified health professionals and that are planned in advance to take place at home. These have been the target of discussions in the media, on social networks, among professional health councils and among the most diverse sectors of society. In this quantitative and descriptive retrospective study, we aim to analyze and describe the results of planned home births attended by a team of obstetric nurses in the city of Campinas, Brazil, from 2013 to 2017. The analyzed records were of women at usual obstetric risk, who had planned home birth attended by a team of obstetric nurses $(n=160)$. Data were obtained by analyzing the medical records of the parturients and the variables analyzed were sociodemographic data, obstetric profile, obstetric and neonatal results. The results showed that $68.75 \%$ of the deliveries lasted up to 8 hours, $53.44 \%$ of the parturients had no perineal lacerations during the expulsion period, the postpartum maternal transfer rate was $2.52 \%$ and only $18.81 \%$ of the parturients needed intervention during the delivery process. Regarding newborns, 97.41\% had a 1st minute Apgar above 8 and 100\% had a 5th minute Apgar above 8. We found that the results are compatible with international studies that consider planned home births as safe as hospital births, in addition to proving the use of the precepts of humanization during childbirth, which respects the woman's choices during the physiological process of giving birth. Based on the analysis of results and comparison with literature data, we can conclude that planned home births with women at habitual obstetric risk do not offer additional risks to traditional hospital births, for both the mother and the baby.
\end{abstract}

Keywords: Home births; Humanization of childbirth assistance; Health information systems; Bioengineering.

\title{
Resumen
}

En las últimas décadas, ha habido un número creciente de partos que son asistidos por profesionales de la salud calificados y que están planificados con anticipación para tener lugar en el hogar. Estos han sido objeto de discusiones en los medios de comunicación, en las redes sociales, entre los consejos profesionales de salud y entre los más diversos sectores de la sociedad. En este estudio retrospectivo cuantitativo y descriptivo, nuestro objetivo es analizar y describir los resultados de partos domiciliarios planificados atendidos por un equipo de enfermeras obstétricas en la ciudad de Campinas, Brasil, de 2013 a 2017. Los registros analizados fueron de mujeres con riesgo obstétrico habitual, que había planificado el parto domiciliario atendido por un equipo de enfermeras obstétricas $(\mathrm{n}=160)$. Los datos se obtuvieron mediante el análisis de las historias clínicas de las parturientas y las variables analizadas fueron datos sociodemográficos, perfil obstétrico, resultados obstétricos y neonatales. Los resultados mostraron que el $68,75 \%$ de los partos duraron hasta 8 horas, el 53,44\% de las parturientas no presentaron laceraciones perineales durante el período de expulsión, la tasa de transferencia maternal posparto fue de 2,52\% y solo el $18,81 \%$ de las parturientas requirieron intervención durante el período del parto. En cuanto a los recién nacidos, el 97,41\% tuvo un Apgar del 1er minuto por encima de 8 y el 100\% tuvo un Apgar del 5o minuto por encima de 8. Encontramos que los resultados son compatibles con estudios internacionales que consideran los partos domiciliarios planificados tan seguros como los partos hospitalarios, además de acreditar el uso de los preceptos de humanización durante el parto, que respeta las elecciones de la mujer durante el proceso fisiológico del parto. A partir del análisis de los resultados y la comparación con los datos de la literatura, podemos concluir que los partos domiciliarios planificados con mujeres en riesgo obstétrico habitual no ofrecen riesgos adicionales a los partos hospitalarios tradicionales, tanto para la madre como para el bebé.

Palabras clave: Partos en casa; Humanización de la asistencia al parto; Sistemas de información sanitaria; Bioingeniería.

\section{Introdução}

A partir da década de 1980, começaram a ser implementadas mudanças nas rotinas obstétricas plasmadas em um modelo humanista de respeito aos direitos das usuárias, substituindo o paradigma hegemônico da alta tecnologia e o abuso de intervenções clínicas, muitas vezes, desnecessárias (Hutton et al., 2014; 2016). Desde então, a temática da humanização do parto e nascimento tem sido reconhecida na prática científica e de direitos femininos (Santos et al., 2015) e tem ocupado espaços sociais e políticos em fóruns científicos de discussão, a partir, principalmente, da mobilização social provocada pelo movimento feminista em prol dos direitos sexuais e reprodutivos das mulheres (The Lancet, 2019).

No entanto, movimentos sociais são fundamentais nesse processo da humanização ao parto e nascimento. Algumas organizações governamentais e não-governamentais (ONGs) como a ReHuNa (Rede pela Humanização do Parto e Nascimento), a "Amigas do Parto" e a rede "Parto do Princípio" são responsáveis pela difusão das recomendações da OMS (Organização Mundial de Saúde) sobre tecnologias apropriadas de assistência obstétrica e neonatal, organização de publicações, eventos e debates, além de formação de opinião objetivando o empoderamento das mulheres (Rattner, 2009). A 
Política Nacional de Humanização do Parto e do Nascimento (PHPN) e a rede cegonha, regulamentada pela portaria $\mathrm{n}^{\circ} 1459$ de 24 de junho de 2011 foram iniciativas do governo federal brasileiro que propuseram um novo modelo de atenção ao parto, nascimento e à saúde da criança com o objetivo de reduzir a morte de mulheres na gravidez e no parto e a mortalidade neonatal. (Ministério da Saúde, 2011).

Os profissionais de saúde também ocupam papel de destaque na humanização do parto, nascimento e na assistência em geral (Ministério da Saúde, 2000; World Health Organization, 2018; Malheiros et al, 2012). Tanto médicos quanto enfermeiros obstetras estão capacitados e autorizados a prestar assistência aos partos de risco habitual e parte destes profissionais reconhece a necessidade da mudança de paradigma, a busca por novos conhecimentos e a atualização da formação (Malheiros et al, 2012). Em 2018, a OMS publicou novas recomendações de cuidados intraparto para uma experiência positiva de nascimento, frente a manutenção de intervenções desnecessárias no parto (World Health Organization, 2018).

A opção pelo local de nascimento tem sofrido modificações nos últimos anos, com aumento dos nascimentos em ambientes não hospitalares como os Centros de Parto Normal ou os partos domiciliares planejados. A assistência ao parto no domicílio não faz parte das políticas públicas brasileiras, mas deve ser informada como opção às gestantes de baixo risco segundo as "Diretrizes Nacionais de Assistência ao Parto Normal" (Ministério da Saúde, 2017).

O parto domiciliar planejado é recomendado pela OMS e aceito pelo Ministério da Saúde desde que obedeça a alguns critérios como gestações de baixo risco, assistência no domicílio prestada por profissionais treinados e presença de um adequado sistema de referência e transporte nos casos de urgência e emergência (Ministério da Saúde, 2017; World Health Organization, 1997).

Em países como Austrália, Inglaterra, Holanda e Canadá, o parto domiciliar é um evento reconhecido e estimulado pelo sistema público de saúde, considerado como modalidade de assistência tão segura quanto o parto hospitalar, menos oneroso ao estado e se mostra como experiência satisfatória às mulheres e familiares. A baixa taxa de morbimortalidade nestes partos está associada a profissionais bem treinados para o atendimento no domicílio e presença de sistema efetivo de transferência e referência (Sanfelice \& Shimo, 2014; de Jonge, et al., 2009; de Jonge, et al., 2013; Birthplace in England Collaborative Group, 2011; Hutton, Reitsma, \& Kaufman, 2009).

Birthplace in England Collaborative Group (2011) e Cheyney et al. (2014) mostram que o parto domiciliar com enfermeiras e/ou parteiras tem menor chance de intervenções, com o mesmo impacto sobre os desfechos perinatais em relação aos partos hospitalares. De Jonge et al. (2013) mostrou que partos domiciliares apresentam menores taxas de morbidade materna aguda grave e Kennare et al. (2010) mostrou que o parto domiciliar planejado tem menores taxas de cesáreas e partos instrumentais.

Nas últimas décadas, tem-se observado um número crescente de nascimentos que são assistidos por profissionais de saúde habilitados e que são planejados antecipadamente para acontecerem em domicílio. Estes têm sido alvo de discussões na mídia, nas redes sociais, entre os conselhos profissionais de saúde e entre os mais diversos setores da sociedade (Sanfelice \& Shimo, 2014). As mulheres que buscam o parto normal domiciliar planejado rompem com seu papel passivo em um modelo hegemônico tecnocrático de atendimento, resgatando para si o próprio parto e o controle sobre seu corpo, com escolhas seguras baseadas em evidências científicas (Frank \& Pelloso, 2013).

Os dados obtidos através dos sistemas de informação em saúde são essenciais para o planejamento e gestão das políticas públicas. Em relação à saúde materna e infantil, destacamos o SisPreNatal e o Sistema de Informações sobre Nascidos Vivos (SINASC). O SINASC é um dispositivo utilizado pelo governo federal com a finalidade de reunir informações epidemiológicas referentes aos nascimentos de todo o território nacional e tem como benefícios subsidiar as intervenções relacionadas à saúde da mulher e da criança para todos os níveis do SUS, além de acompanhar a evolução dos indicadores. Tal 
ferramenta permite a identificação de prioridades de intervenção, o que contribui para efetiva melhoria do sistema (Ministério da Saúde, 2021).

Os dados que alimentam o SINASC são obtidos através da Declaração de Nascidos Vivos (DN), documento numerado pelo Ministério da Saúde, em três vias, padronizado em todo o país e distribuído aos serviços de saúde e cartórios. Segundo a Lei $\mathrm{n}^{\circ}$ 12.662, a Declaração de Nascido Vivo deverá ser emitida pelo profissional de saúde responsável por acompanhar a gestação, o parto ou o recém-nascido, inscrito no Cadastro Nacional de Estabelecimentos de Saúde (CNES) ou no respectivo conselho profissional (Ministério da Saúde, 2012a). Os principais dados obtidos através da DN fornecem indicadores de saúde sobre o pré-natal, assistência ao parto, vitalidade ao nascer, mortalidade infantil e materna.

Dados do SINASC mostram que em 2006 houve 937 partos domiciliares dentre um total de 603.368 nascimentos no estado de São Paulo, correspondendo a 0,15\%. Dados de 2016 mostram porcentagem de 0,32\% (1.924 partos domiciliares em um total de 601.239), mostrando nesta década o aumento no número absoluto e relativo deste tipo de parto (Ministério da Saúde, 2021). A insuficiência de dados sobre os partos domiciliares planejados (Koettker, Bruggemann, \& Knobel, 2017) não permite uma adequada avaliação deste modelo de assistência no Brasil, tornando difícil avaliar riscos e benefícios para mães e bebês.

Conhecer a realidade e as características em que os partos e nascimentos acontecem no Brasil é uma etapa importante para a implementação de mudanças sobre a assistência ao parto. Dessa forma, essa pesquisa tem como principal objetivo analisar e descrever os resultados maternos dos partos domiciliares planejados atendidos por uma equipe de enfermeiras obstetras da cidade de Campinas-SP no período de 2013 a 2017.

\section{Metodologia}

Trata-se de um estudo retrospectivo quantitativo e descritivo (Gauch Jr, 2015) dos atendimentos realizados por uma dupla de enfermeiras obstétricas que atuam conjuntamente desde 2012 na região de Campinas-SP, a equipe Arte de Nascer. Atualmente a equipe presta assistência às famílias durante a gestação, trabalho de parto, parto e pós-parto, mediante pagamento privado, com foco no parto domiciliar.

São elegíveis ao parto domiciliar apenas mulheres que estejam realizando adequado acompanhamento pré-natal e com gestação de baixo risco, ou seja "em que não há necessidade de se utilizar alta densidade tecnológica em saúde e nos quais a morbidade e a mortalidade materna e perinatal são iguais ou menores do que as da população em geral" (Ministério da Saúde, 2012b).

Foram coletados dos prontuários das parturientes os seguintes dados: dados demográficos, perfil obstétrico, resultados obstétricos e neonatais. O estudo foi submetido e aprovado pelo Comitê de Ética em Pesquisa (CEP) da Universidade Brasil sob parecer número 2.082.388 e segue os princípios da Declaração de Helsinque para pesquisa que envolvem seres humanos.

Foram estudadas mulheres de risco obstétrico habitual, que tiveram parto domiciliar planejado atendido pela equipe Arte de Nascer, no estado de São Paulo, no período de fevereiro de 2013 a fevereiro de 2017 (n=160). Foram excluídos do estudo os prontuários de mulheres que vivenciaram o parto domiciliar de forma acidental ou assistida por profissionais não pertencentes a equipe Arte de Nascer, gestantes que iniciaram o pré-natal com a equipe, mas devido ao risco obstétrico não habitual, foram excluídas do parto domiciliar, além de mulheres que iniciaram o trabalho de parto no domicílio, mas precisaram ser transferidas para o hospital $(\mathrm{n}=31)$. Obteve-se um total de 116 prontuários incluídos no estudo. Os dados sociodemográficos coletados foram idade e escolaridade no momento do parto, enquanto os resultados obstétricos pesquisados foram: estado das membranas no início do trabalho de parto, uso de técnicas não farmacológicas, alimentação, presença de pessoas externas à equipe, local/posição do parto, duração do trabalho de parto em horas, condições do períneo, dequitação da 
placenta, intercorrências intra e pós-parto, e necessidade de transferência.

Para o processamento e a análise dos dados foi utilizado o software Excel (Microsoft@), com análise descritiva e apresentação das frequências absolutas e relativas. Os dados estão apresentados em tabelas e figuras.

\section{Resultados}

Pela análise do perfil sociodemográfico observamos uma idade média das parturientes de 30,67 anos, entre 18 e 40 anos com desvio padrão (DP) de 3,87. A escolaridade da maioria $(94,54 \%)$ foi acima de 12 anos de estudo, ou seja, ensino superior completo ou incompleto.

Os resultados obstétricos foram os seguintes: $53,44 \%$ das parturientes $(n=62)$ iniciou o trabalho de parto com membranas íntegras e 22,41\% ( $\mathrm{n}=26$ ) com bolsa rota de até 24 horas. A duração média do trabalho de parto foi de 6,87 horas, variando de 1 a 34 horas, sendo que em 68,75\% dos casos o trabalho de parto teve duração de até 8 horas (n=66). A maioria das mulheres $(58 \%)$ se alimentou durante o trabalho de parto, conforme mostra a Figura 1. Parceiro e doula foram as principais pessoas externas à equipe durante o trabalho de parto (Figura 2).

Figura 1. Alimentação durante o trabalho de parto nos atendimentos da Equipe Arte de Nascer.

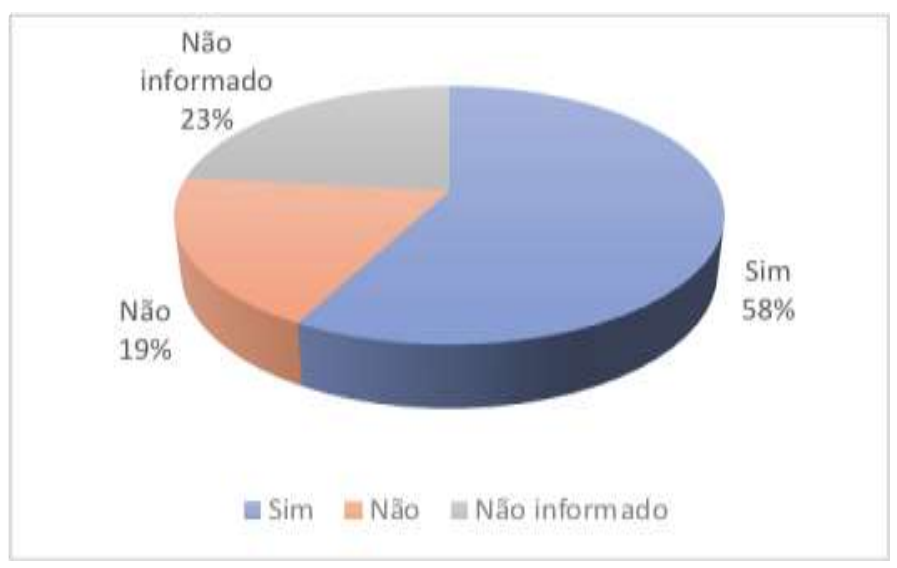

Fonte: Autores (2021).

Figura 2. Pessoas externas à equipe durante o trabalho de parto durante o trabalho de parto nos atendimentos.

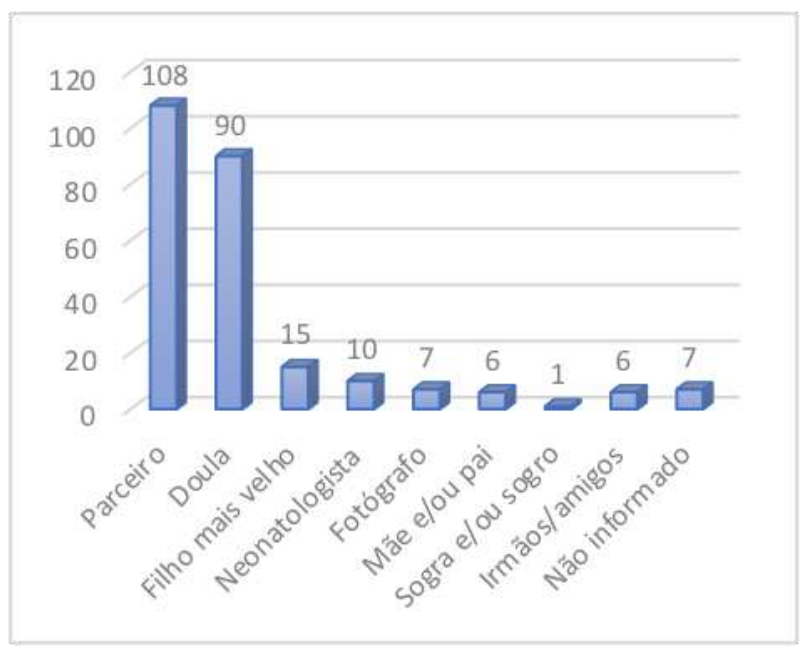

Fonte: Autores (2021).

A banheira ou piscina $(n=47)$ e a banqueta de parto $(n=38)$ foram os locais mais utilizados pelas parturientes no 
Research, Society and Development, v. 10, n. 12, e234101220358, 2021

(CC BY 4.0) | ISSN 2525-3409 | DOI: http://dx.doi.org/10.33448/rsd-v10i12.20358

momento do nascimento e as principais posições utilizadas foram quatro apoios (n=23) e cócoras (n=18) (Figura 3).

Figura 3. Local/posição do parto durante o trabalho de parto nos atendimentos da Equipe Arte de Nascer.

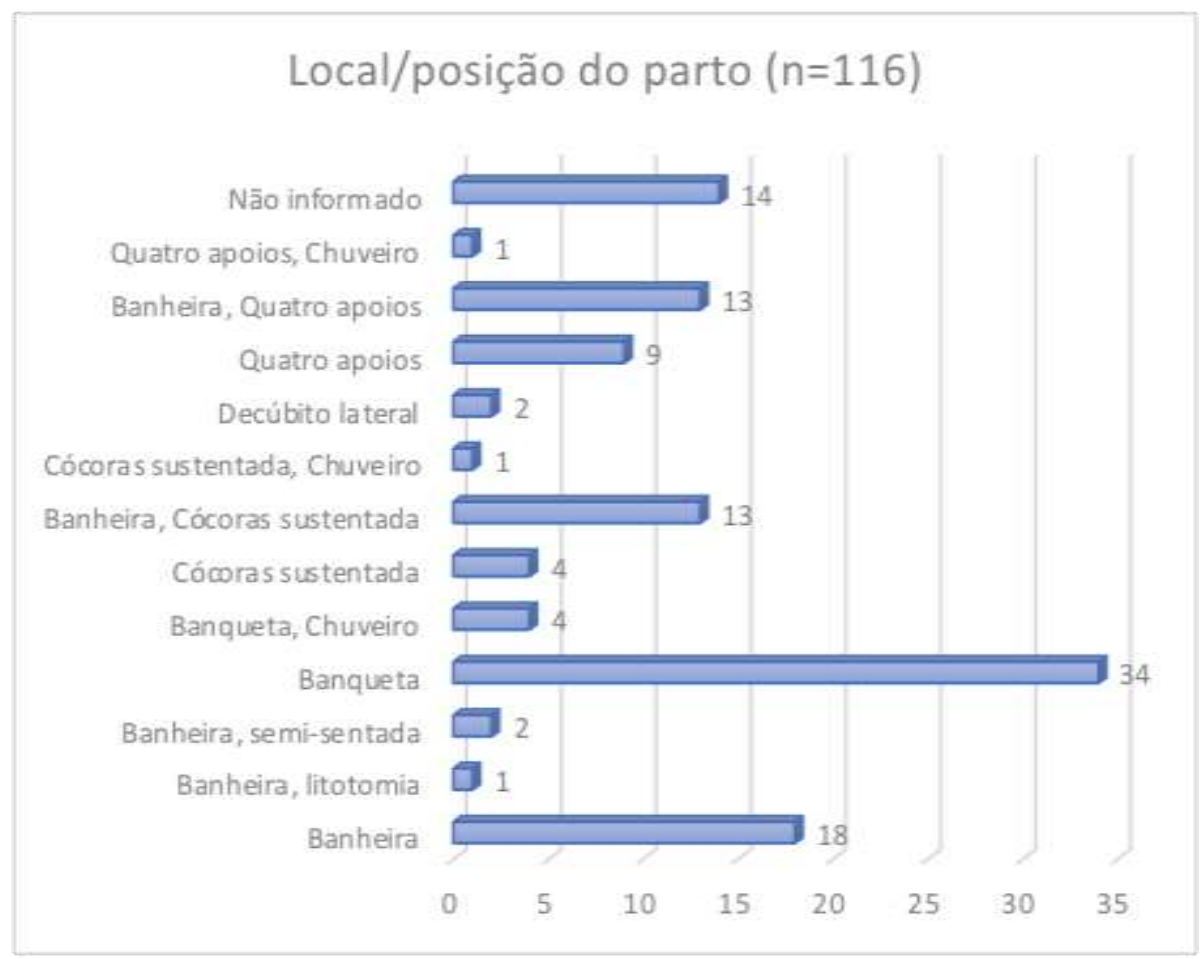

Fonte: Autores (2021).

As técnicas não farmacológicas mais utilizadas neste processo foram piscina $(57,75 \%)$, chuveiro $(56,89 \%)$ e bola de Pilates $(31,89 \%)$ (Figura 4).

Figura 4. Uso de técnicas não farmacológicas durante o trabalho de parto nos atendimentos da Equipe Arte de Nascer.

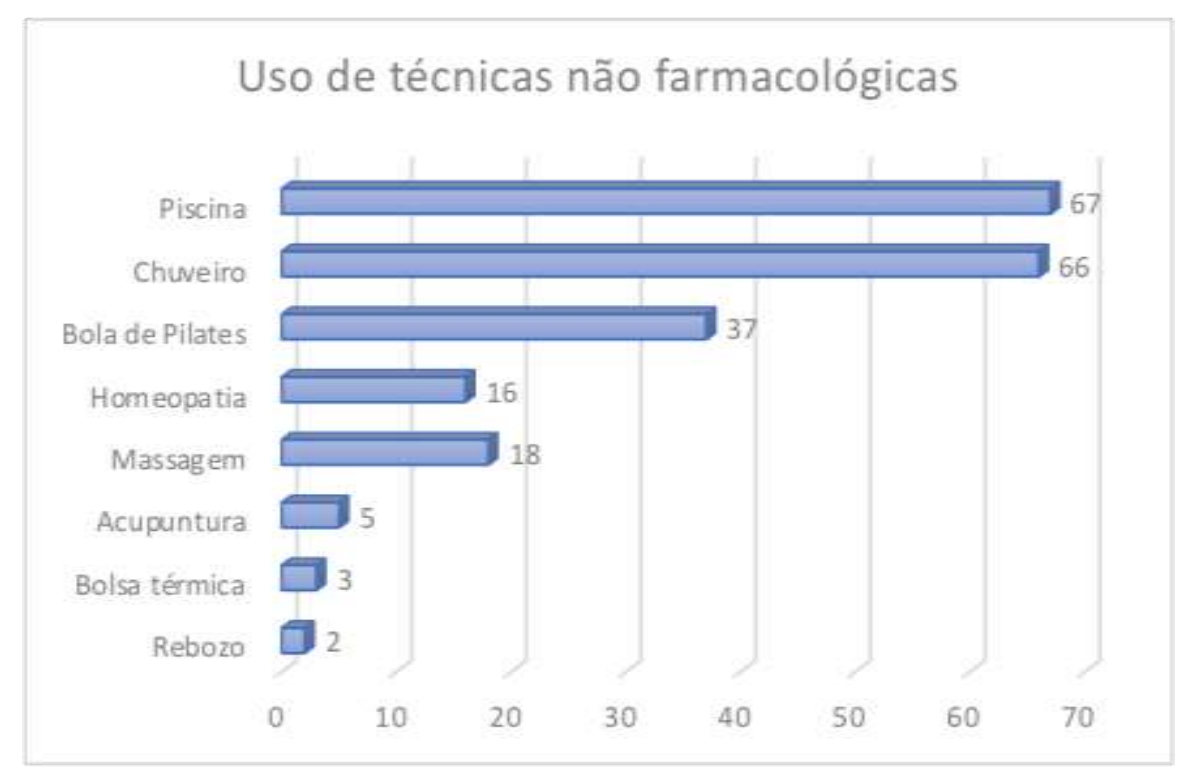

Fonte: Autores (2021).

Grande parte das parturientes $(53,44 \%, \mathrm{n}=62)$ não teve lacerações de períneo durante o período expulsivo, ou 
Research, Society and Development, v. 10, n. 12, e234101220358, 2021

(CC BY 4.0) | ISSN 2525-3409 | DOI: http://dx.doi.org/10.33448/rsd-v10i12.20358

apresentou lacerações que não necessitaram sutura (Figura 5).

Figura 5. Condições do períneo nos atendimentos.

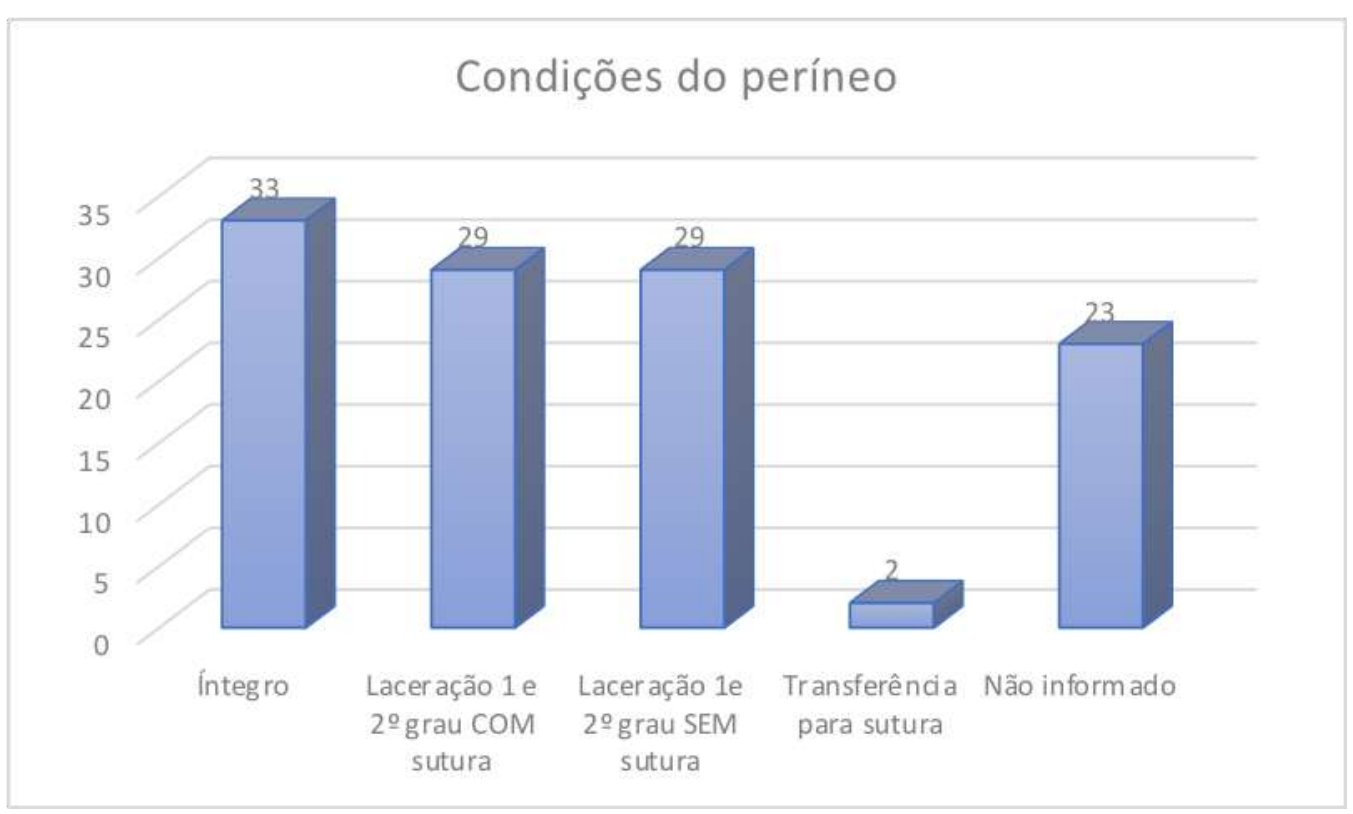

Fonte: Autores (2021).

A taxa de transferência materna pós-parto foi de 2,52\% (Figura 6) e apenas $18,81 \%$ das parturientes necessitaram de intervenções durante o processo do parto, sendo a injeção de Ocitocina via intramuscular o procedimento mais utilizado ( $\mathrm{n}=16$, 13,68\%) (Figura 7).

Figura 6. Transferências pós-parto - motivos nos atendimentos.

\section{Transferências pós-parto - motivos}

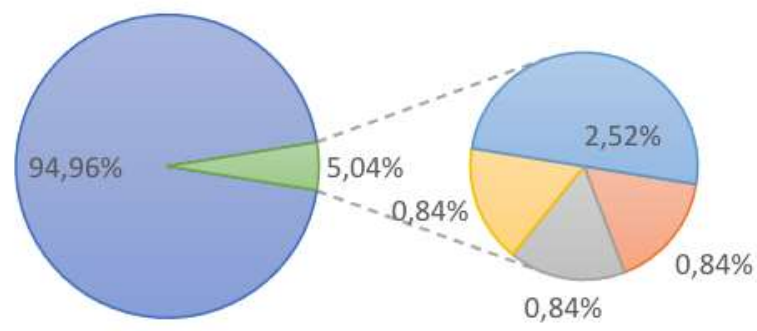


Figura 7. Intervenções no trabalho de parto, parto e pós-parto imediato nos atendimentos da Equipe Arte de Nascer.

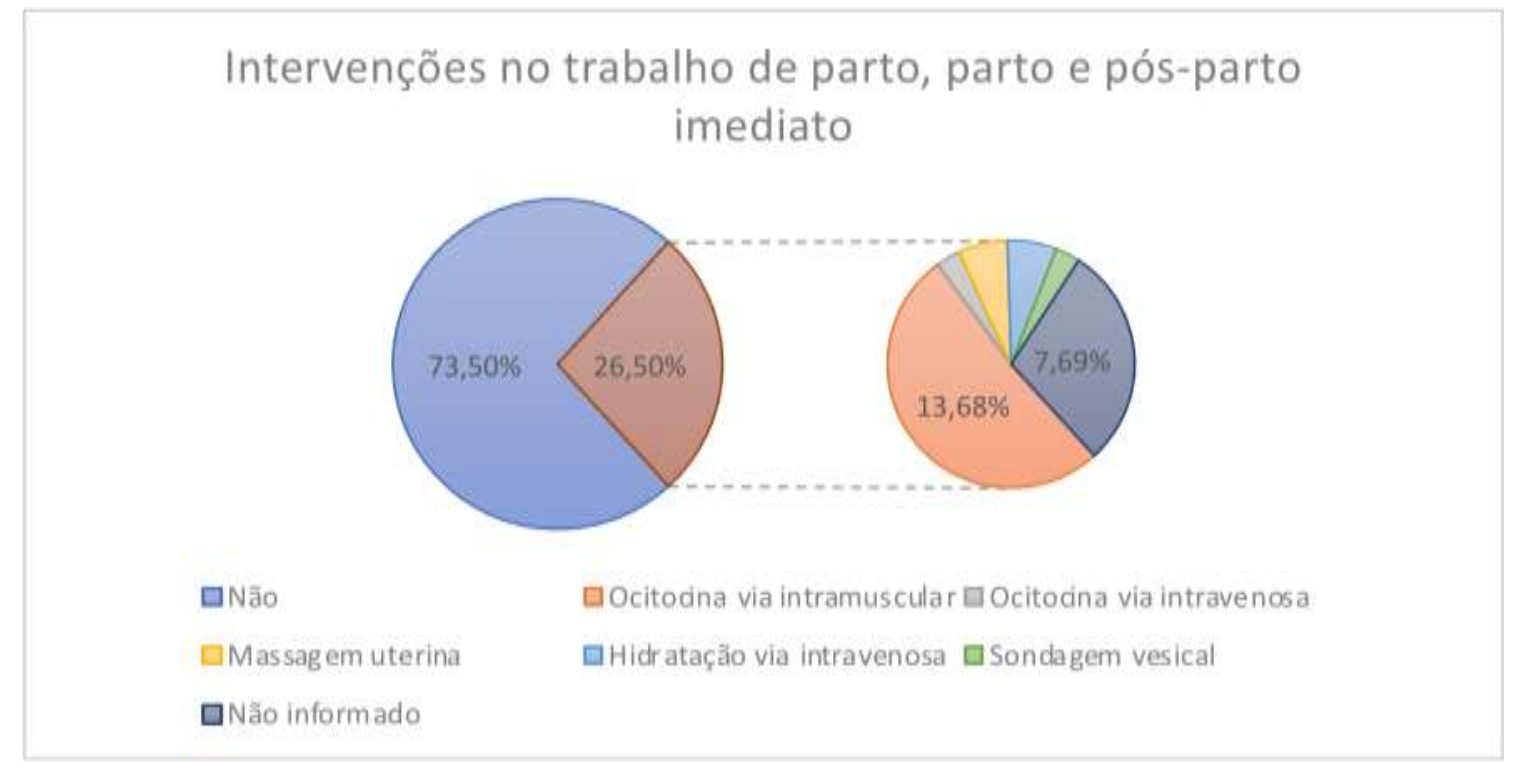

Fonte: Autores (2021).

Em relação aos dados neonatais, verificado predomínio de Apgar de $1^{\circ}$ minuto acima 8 (97,41\%), e $100 \%$ dos casos com Apgar de $5^{\circ}$ minuto satisfatório, ou seja, acima de 8. Apenas dois neonatos necessitaram de intervenções logo após o nascimento (Tabela 1).

Tabela 1. Apgar de $1^{\circ}$ e $5^{\circ}$ minutos, necessidades de intervenção nos neonatos atendidos pela equipe.

\begin{tabular}{|c|c|c|}
\hline Variáveis & $\mathbf{n}$ & Porcentagem \\
\hline \multicolumn{3}{|l|}{ Apgar de $1^{\circ}$ minuto } \\
\hline Menor 7 & 3 & $2,58 \%$ \\
\hline Maior ou igual a 7 & 113 & $97,41 \%$ \\
\hline \multicolumn{3}{|l|}{ Apgar de $5^{\circ}$ minuto } \\
\hline Menor 7 & 0 & 0 \\
\hline Maior ou igual a 7 & 116 & $100 \%$ \\
\hline \multicolumn{3}{|c|}{ Necessidade de intervenção } \\
\hline Não informado & 8 & $6,89 \%$ \\
\hline Não & 106 & $91,37 \%$ \\
\hline Sim & 2 & $1,72 \%$ \\
\hline Máscara de oxigênio & 1 & $0,86 \%$ \\
\hline Reanimação neonatal & 1 & $0,86 \%$ \\
\hline
\end{tabular}

Fonte: Autores (2021).

\section{Discussão}

O perfil sociodemográfico evidenciou faixa etária predominante entre 31 e 40 anos, com poucas parturientes abaixo de 20 anos. Em relação ao processo de reprodução humana, registra-se uma tendência cada vez maior de gravidez entre mulheres que se encontram na faixa dos 35 anos (Caetano, Netto, \& Manduca, 2011; Pinheiro et al., 2019) provavelmente decorrente da mudança nos padrões familiares e características da natalidade, com diminuição progressiva das taxas globais e adiamento da gravidez planejada (Schupp, 2006).

Observamos que a maioria das gestantes atendidas pela equipe de enfermeiras tem mais de 12 anos de escolaridade (90\%), ou seja, ensino superior incompleto ou completo. As variáveis idade e escolaridade elevadas, são condizentes com 
estudos nacionais e internacionais (Koettker, Bruggemann, \& Knobel, 2017), e em geral estão relacionadas com maior acesso aos meios de informação, possibilidade de opção e uso do direito à escolha informada (Colacioppo et al., 2010).

Durante o trabalho de parto pudemos observar nesta pesquisa a liberdade das mulheres em relação à escolha dos acompanhantes: o parceiro estava presente em $99 \%$ dos casos com registro em prontuário e 82,56\% tinham uma doula durante o processo para apoio físico e/ou emocional. Desde 2005 temos a Lei federal $n^{\circ} 11.108$ que obriga os serviços públicos, da rede própria ou conveniada, permitir à todas as mulheres um acompanhante de sua escolha durante o pré-natal e todas as fases de internação para o parto, incluindo os períodos do trabalho de parto, parto e pós-parto (Brasil, 2005). Uma revisão sistemática recente conclui que este apoio contínuo durante o trabalho de parto pode aumentar as taxas de parto vaginal espontâneo, diminuir a duração do trabalho de parto, o número de nascimentos por cesariana, o uso de analgesia, diminuir as taxas de baixo Índice de Apgar de $5^{\circ}$ minuto, além de auxiliar na diminuição dos sentimentos negativos sobre a experiência de parto (Bohren et al., 2017). No entanto, o inquérito "Nascer no Brasil" evidenciou que 24,5\% das mulheres não teve qualquer acompanhante e 55,2\% tiveram acompanhante parcialmente durante a internação hospitalar. Dentre aquelas que não tiveram acompanhante, a principal razão foi a proibição do hospital e apenas 1,4 \% do número total de mulheres não desejava um acompanhante (Diniz et al., 2014).

Na maior parte dos casos aqui levantados as parturientes utilizaram técnicas não farmacológicas para alívio da dor, principalmente chuveiro e piscina, além de se alimentaram durante o trabalho de parto. Estes dados vão de encontro às últimas diretrizes nacionais de assistência ao parto, que preconizam uso de massagem, chuveiro e outros métodos para alívio da dor, além de liberar uma alimentação leve àquela parturiente que não está com risco imediato de realizar procedimento cirúrgico (Ministério da Saúde, 2017). Quando falamos de partos hospitalares, levantamento nacional verificou que menos de um terço do grupo de risco obstétrico habitual se alimentou durante o trabalho de parto e utilizou procedimentos não farmacológicos para alívio da dor (Leal et al., 2014).

As gestantes estudadas neste trabalho estiveram livres para optar pela posição e local de nascimento, sendo a banheira e a banqueta de parto os locais mais utilizados e as posições de quatro apoios e cócoras identificadas como as preferidas durante o expulsivo. As gestantes são incentivadas a procurar uma posição que lhe permita a melhor experiência possível, com preferência às posições verticalizadas que reduzem o tempo de trabalho do parto (Ministério da Saúde, 2017). No inquérito sobre os partos hospitalares, até $90 \%$ das mulheres estavam em posição de litotomia durante o nascimento (Leal et al., 2014).

Um dado importante em relação ao parto atendido pelas enfermeiras obstétricas foi a ausência de episiotomia, procedimento que não costuma ser realizado nos partos domiciliares. Apenas 31,18\% das parturientes necessitaram de sutura após lacerações de primeiro ou segundo grau no períneo. Este número se contrapõe à quantidade de episiotomias realizadas nos hospitais, segundo o "Nascer no Brasil", que mostrou índices de 56,1\% nas gestantes de risco obstétrico habitual (Leal et al., 2014). Uma revisão sistemática sobre o uso rotineiro da episiotomia mostrou que ela aumenta a chance de trauma perineal, o número de suturas e as complicações na cicatrização, e não tem diferença em relação às lacerações espontâneas quando se avalia a dor no local, incontinência urinária ou dispareunia (Carroli \& Mignini, 2014). As "Diretrizes Nacionais de Assistência ao Parto Normal" também orientam que a episiotomia não deve ser realizada rotineiramente no parto normal espontâneo (Ministério da Saúde, 2017).

Verificando os dados sobre procedimentos realizados pela equipe durante o processo do nascimento, observamos que $80,37 \%$ das parturientes não foi submetida a qualquer intervenção. Ao comparar com o levantamento "Nascer no Brasil", verificamos que apenas 5,6\% das parturientes de risco habitual pariram sem qualquer tipo de intervenção na fisiologia do trabalho de parto (Leal et al., 2014). Em revisão sistemática foi demonstrado que, entre estudos cuidadosamente selecionados de mulheres com gravidez de baixo risco em países de alta renda, o local planejado de parto parece ter pouco impacto significativo sobre os resultados perinatais adversos. Além disso, as mulheres que planejaram dar à luz em um centro de parto 
ou em casa tiveram chances significativamente menores de intervenção e morbidade grave no trabalho de parto e parto (Scarf et al., 2019).

As transferências hospitalares eletivas ou a pedido da parturiente e família corresponderam a 8,12\% dos casos, enquanto estudo semelhante no Sul do Brasil evidenciou taxa de 14,3\% (Colacioppo et al., 2010). As parturientes que precisaram de transferência ao hospital devido intercorrências obstétricas durante ou logo após o trabalho de parto totalizaram 6,25\%, compatível com os estudos nacionais disponíveis que mostram variação entre 5,7\% e 7,4\% (World Health Organization, 2018; Malheiros et al., 2012). Revisão sistemática sobre o tema mostra taxas de transferências variando de 9,9\% a 31,9\%, mas não diferencia se houve indicação obstétrica ou a pedido da paciente e/ou familiares (Blix et al., 2014). A taxa de cesárea dentre as pacientes que foram atendidas pela equipe no domicílio, mas tiveram os partos ocorrendo no hospital, foi de 5,62\%, mas em 3,75\% dos prontuários não havia o registro em relação ao desfecho. Mesmo assim, os dados são compatíveis com as orientações da Organização Mundial da Saúde que preconiza até 15\% de partos cirúrgicos (Carniel, Zanolli, \& Morcillo, 2007).

O desfecho satisfatório evidenciado pelo Apgar de $5^{\circ}$ minuto dos neonatos, mostra-se como um dos pontos que reforça a segurança do parto domiciliar planejado, assim como evidenciado em outros estudos deste tipo. Poucos bebês deste levantamento necessitaram de alguma intervenção logo após o nascimento, e todos tiveram recuperação completa após os procedimentos realizados. Verificamos que estes dados são compatíveis com estudos internacionais (de Jonge et al., 2009; de Jonge et al., 2013; Birthplace in England Collaborative Group, 2011; Hutton, Reitsma, \& Kaufman, 2009; Cheyney et al., 2014; Kennare et al., 2010; Janssen et al., 2009) que consideram o parto domiciliar planejado tão seguro quanto o hospitalar. Além de comprovar a utilização dos preceitos da humanização ao parto, que respeita as escolhas da mulher durante o processo fisiológico de parir. Observamos o trabalho da equipe condizente com as boas práticas na atenção ao trabalho de parto e parto, preconizados pelo Ministério da Saúde (Ministério da Saúde, 2017) e OMS (World Health Organization, 2018; World Health Organization, 1997). Em revisão sistemática, evidências sobre gestações de baixo risco indicam que o local de nascimento não teve impacto estatisticamente significativo na mortalidade infantil (Scarf et al., 2019). E ainda, que as menores chances de morbidade materna e intervenção obstétrica apoiam a expansão de centros de parto e opções de partos em casa para mulheres com gestações de baixo risco (Scarf et al., 2019; Zielinski et al., 2015).

$\mathrm{Na}$ análise dos prontuários estudados, foi verificado uma quantidade importante de subregistros em relação as variáveis avaliadas neste estudo, chegando a até $23,27 \%$ de respostas "não informado". A coleta retrospectiva dos dados teve destaque como limitação deste estudo, uma vez que o não preenchimento completo dos prontuários limitou a análise de algumas variáveis, principalmente aquelas relacionadas ao trabalho de parto, como presença de outras pessoas durante o processo, técnicas utilizadas para alívio da dor ou alimentação durante o trabalho de parto.

\section{Conclusão}

Este estudo contribui para o conhecimento de dados nacionais e auxilia no reconhecimento do domicílio como local de nascimento pelos profissionais de saúde que ainda possuem resistência, além de reforçar a segurança do parto domiciliar planejado em gestantes de risco obstétrico habitual. Pela análise dos resultados e comparação com dados da literatura, podemos concluir que o parto domiciliar planejado com mulheres de risco obstétrico habitual não oferece riscos adicionais ao parto tradicional em hospital, tanto para a parturiente quanto para o bebê.

Trabalhos futuros com ampliação da avaliação de prontuários e comparação com um grupo neutro de partos hospitalares devem ser realizados a fim de possibilitar respaldo estatístico para algumas análises junto às informações fornecidas pelo SINASC em relação aos partos domiciliares. Pois as informações fornecidas pelo SINASC, não diferenciam os 
partos domiciliares planejados dos partos domiciliares acidentais, o que pode levar a conclusões erradas ao unificar estas duas modalidades.

\section{Referências}

Birthplace in England Collaborative Group. (2011). Perinatal and maternal outcomes by planned place of birth for healthy women with low risk pregnancies: the Birthplace in England national prospective cohort study. BMJ. 343:d7400. 10.1136/bmj.d7400

Blix, E., Kumle, M., Kjærgaard, H., Øian, P., \& Lindgren, H. E. (2014). Transfer to hospital in planned home births: a systematic review. BMC Pregnancy and Childbirth, 14(179), 1-11.

Bohren, M., Hofmeyr, G., Sakala, C., Fukuzawa, R., \& Cuthbert, A. (2017). Continuous support for women during childbirth. Cochrane Database Syst Rev.(7). Art. No.: CD003766. 10.1002/14651858.CD003766.pub6

Brasil. (2005). Lei n 11.108, de 7 de abril de 2005 (altera a lei n 8.080, de 19 de setembro de 1990). Do subsistema de acompanhamento durante o trabalho de parto, parto e pós-parto imediato.

Caetano, L. C., Netto, L., \& Manduca, J. d. (2011). Gravidez depois dos 35 anos: uma revisão sistemática da literatura. Rev. Min. Enferm., 15 (4), $579-587$.

Carniel, E. d., Zanolli, M., \& Morcillo, A. M. (2007). Fatores de risco para indicação do parto cesáreo em Campinas (SP). Rev. Bras. Ginecol. Obstet., 29(1), 34-40.

Carroli, G., \& Mignini, L. (2014). Episiotomy for vaginal birth. Cochrane Database Syst Rev. (11), 1-53.

Cheyney, M., Bovbjerg, M., Everson, C., Gordon, W., Hannibal, D., \& Vedam, S. (2014). Outcomes of care for 16,924 planned home births in the United States: the Midwives Alliance of North America Statistics Project, 2004 to 2009. J Midwifery Womens Health, 59(1), 17-27.

Colacioppo, P., Koiffman, M., Riesco, M. G., Schneck, C., \& Osava, R. (2010). Parto domiciliar planejado: resultados maternos e neonatais. Rev. Enferm. Referencia, III(2), 81-90.

de Jonge, A., Mesman, J. A., Mannien, J., Zwart, J. J., van Dillen, J., \& van Rossmalen, J. (2013). Severe adverse maternal outcomes among low risk women with planned home versus hospital births in the Netherlands: nationwide cohort study. BMJ 346: f3263

de Jonge, A., van der Goes, B. Y., Ravelli, A. C., Amelink-Verburg, M. P., Mol, B. W., Nijhuis, J. G., Bennebroek Gravenhorst, J., \& Buitendijk, S. E. (2009). Perinatal mortality and morbidity in a nationwide cohort of 529,688 low-risk planned home and hospital births. BJOG: an international journal of obstetrics and gynaecology, 116(9), 1177-1184. https://doi.org/10.1111/j.1471-0528.2009.02175.x

Diniz, C., d Orsi, E., Domingues, R., Torres, J., Dias, M., Schneck, C., \& Sandall, J. (2014). Implementação da presença de acompanhantes durante a internação para o parto: dados da pesquisa nacional Nascer no Brasil. Cad. Saúde Pública, 30(1), S140-S153.

Frank, T. C., \& Pelloso, S. (2013). The perception of professionals regarding planned home birth. Rev. Gaucha Enferm., 34(1), 22-29.

Gauch Jr, H. (2015). Scientific Method in Practice. Cambridge: Cambridge University Press. 10.1017/CBO9780511815034

Hutton, E. K., Cappelletti, A., Reitsma, A. H., Simioni, J., Horne, J., McGregor, C., \& Ahmed, R. J. (2016). Outcomes associated with planned place of birth among women with low-risk pregnancies. CMAJ: Canadian Medical Association journal = journal de l'Association medicale canadienne, 188(5), E80-E90. https://doi.org/10.1503/cmaj.150564

Hutton, E. K., Reitsma, A., \& Kaufman, K. (2009). Outcomes associated with planned home and planned hospital births in low-risk women attended by midwives in Ontario, Canada, 2003-2006: a retrospective cohort study. Birth, 36(3), 180-189.

Hutton, E. K., Reitsma, A., Thorpe, J., Brunton, G., \& Kaufman, K. (2014). Protocol: systematic review and meta-analyses of birth outcomes for women who intend at the onset of labour to give birth at home compared to women of low obstetrical risk who intend to give birth in hospital. Systematic reviews, 3 , 55 . https://doi.org/10.1186/2046-4053-3-55

Janssen, P., Saxell, L., Page, L., Klein, M., Liston, R., \& Lee, S. (2009). Outcomes of planned home birth with registered midwife versus planned hospital birth with midwife or physician. CMAJ, 181(6-7), 377-383.

Kennare, R., Keirse, M., Tucker, G., \& Chan, A. (2010). Planned home and hospital births in South Australia, 1991-2006: differences in outcomes. Med J Aust., 192(2), 76-80.

Koettker, J. G., Bruggemann, O., \& Knobel, R. (2017). Resultados maternos dos partos domiciliares planejados assistidos por enfermeiras da equipe Hanami no sul do Brasil, 2002-2012. Texto Contexto Enferm, 26(1), 1-11.

Leal, M., Pereira, A. E., Domingues, R. S., Theme Filha, M. M., Dias, M. B., Nakamura-Pereira, M., \& da Gama, S. G. (2014). Intervenções obstétricas durante o trabalho de parto e parto em mulheres brasileiras de risco habitual. Cad. Saúde Pública, 30(1), S17-S47.

Malheiros, P. A., Alves, V. H., Rangel, T. S., \& Vargens, O. M. (2012). Parto e nascimento: Saberes e práticas humanizadas. Texto Contexto Enferm, 21(2), 329-337.

Ministério da Saúde. (2000). Instituir o Programa de Humanização no Pré-natal e Nascimento, no âmbito do Sistema Único de Saúde. Ministério da Saúde, Portaria GM/MS n.569. 
Research, Society and Development, v. 10, n. 12, e234101220358, 2021

(CC BY 4.0) | ISSN 2525-3409 | DOI: http://dx.doi.org/10.33448/rsd-v10i12.20358

Ministério da Saúde. (2011). Portaria nº 1.459, de 24 de junho de 2011. Institui, no âmbito do Sistema Único de Saúde a Rede Cegonha. Gabinete do Ministro. http://bvsms.saude.gov.br/bvs/saudelegis/gm/2011/prt1459_24_06_2011.html.

Ministério da Saúde. (2012a). Lei $\mathrm{n}^{\circ}$ 12.662, de 5 de junho de 2012. Assegura validade nacional à Declaração de Nascido Vivo - DNV. http://www.planalto.gov.br/ccivil_03/_ato2011-2014/2012/lei/112662.htm

Ministério da Saúde. (2012b). Cadernos de Atenção Básica: atenção ao pré-natal de baixo risco. Série A. Normas e Manuais Técnicos Cadernos de Atenção Básica, $n^{\circ}$ 32. Disponível em: https://bvsms.saude.gov.br/bvs/publicacoes/cadernos_atencao_basica_32_prenatal.pdf

Ministério da Saúde. (2017). Diretrizes nacionais de assistência ao parto normal: versão resumida. http://bvsms.saude.gov.br/bvs/publicac oes/diretrizes_nacionais_assistencia_parto_normal.pdf

Ministério da Saúde. (2021). SINASC-Sistema de Informações de Nascidos Vivos. http://sinasc.saude.gov.br/

Pinheiro, R. L., Areia, A. L., Pinto, A. M., \& Donato, H. (2019) Advanced Maternal Age: Adverse Outcomes of Pregnancy, A Meta-Analysis. Acta Med Port 32(3):219-226

Rattner, D. (2009). Humanização na atenção a nascimentos e partos: ponderações sobre políticas públicas. Interface (Botucatu), 13(1), 759-768.

Sanfelice, C. F., \& Shimo, A. K. (2014). Home childbirth: progress or retrocession? Rev. Gaúcha Enferm., 35(1), 157-160.

Santos, R. A. A. dos, Melo, M. C. P. de, \& Cruz, D. D. (2015) Trajetória de humanização do parto no brasil a partir de uma revisão integrativa de literatura. Caderno de Cultura e Ciência, Ano IX, v.13, n.2. http://dx.doi.org/10.14295/cad.cult.cienc.v13i2.838

Scarf, V. L., Rossiter, C., Vedam, S., Dahlen, H. G., Ellwood, D., Forster, D., et al. (2018) Maternal and perinatal outcomes by planned place of birth among women with low-risk pregnancies in high-income countries: a systematic review and meta-analysis. Midwifery. 62:240-55. https://doi.org/10.1016/j.midw.2018.03.024

Schupp, T. (2006). Gravidez após os 40 anos de idade: análise dos fatores prognósticos para resultados maternos e perinatais adversos. PhD thesis - USP.

The Lancet (2019). The erosion of women's sexual and reproductive rights. Lancet (London, England), 393(10183), 1773. https://doi.org/10.1016/S0140$6736(19) 30990-0$

World Health Organization. (1997). Care in Normal Birth: A Practical Guide. Birth 24(2)

World Health Organization. (2018). Intrapartum care for a positive childbirth experience. http://apps.who.int/iris/bitstream/handle/10665/2 60178/9789241550215-eng.pdf

Zielinski, R., Ackerson, K., \& Kane Low, L. (2015). Planned home birth: benefits, risks, and opportunities. International Journal of Women's Health, 7, pp. $361-377$ 